\title{
Genetic variation of Glucose Transporter-1 (GLUT1) and albuminuria in 10,278 European Americans and African Americans: a case-control study in the Atherosclerosis Risk in Communities (ARIC) Study
}

Charles C Hsu ${ }^{1,6}$, Wenhong L Kao ${ }^{1}$, Michael W Steffes ${ }^{2}$, Tejal Gambir ${ }^{1}$, Frederick L Brancati ${ }^{1}$, Charles W Heilig ${ }^{1,5}$, Alan R Shuldiner ${ }^{3}$, Eric A Boerwinkle ${ }^{4}$, Josef Coresh $^{1 *}$

\begin{abstract}
Background: Evidence suggests glucose transporter-1(GLUT1) genetic variation affects diabetic nephropathy and albuminuria. Our aim was to evaluate associations with albuminuria of six GLUT1 single nucleotide polymorphisms (SNPS), particularly Xbal and the previously associated Enhancer-2(Enh2) SNP.

Methods: A two-stage case-control study was nested in a prospective cohort study of 2156 African Americans and 8122 European Americans with urinary albumin-to-creatinine ratio(ACR). Cases comprised albuminuria( $N=825 ; \geq$ $30 \mu \mathrm{g} / \mathrm{mg})$ and macroalbuminuria( $\mathrm{N}=173 ; \geq 300 \mu \mathrm{g} / \mathrm{mg})$. ACR $<30 \mu \mathrm{g} / \mathrm{mg}$ classified controls $(\mathrm{n}=9453)$. Logistic regression and odds ratios(OR) assessed associations. The evaluation phase(stage 1, $\mathrm{n}=2938$ ) tested associations of albuminuria( $\mathrm{n}=305)$ with six GLUT1 SNPs: rs841839, rs3768043, rs2297977, Enh2(rs841847) Xbal(rs841853), and rs841858. Enh2 was examined separately in the replication phase(stage $2, n=7340$ ) and the total combined sample $(n=10,278)$, with all analyses stratified by race and type 2 diabetes.

Results: In European Americans, after adjusting for diabetes and other GLUT1 SNPs in stage 1, Enh2 risk genotype (TT) was more common in albuminuric cases $(\mathrm{OR}=3.37, \mathrm{P}=0.090)$ whereas Xbal $(\mathrm{OR}=0.94, \mathrm{p}=0.931)$ and remaining SNPs were not. In stage 1, the Enh2 association with albuminuria was significant among diabetic European Americans $(\mathrm{OR}=2.36, \mathrm{P}=0.025)$. In African Americans, Enh2 homozygosity was rare(0.3\%); Xbal was common(18.0\% AA) and not associated with albuminuria. In stage 2( $n=7,340)$, Enh2 risk genotype had increased but non-significant $O R$ among diabetic European Americans $(\mathrm{OR}=1.66, \mathrm{P}=0.192)$ and not non-diabetics $(\mathrm{OR}=0.99$, $\mathrm{p}=0.953)$, not replicating stage 1. Combining stages 1 and 2, Enh2 was associated with albuminuria(OR 2.14 [1.203.80], $P=0.009)$ and macroalbuminuria(OR 2.69, [1.02-7.09], $P=0.045)$ in diabetic European Americans. The Enh2 association with macroalbuminuria among non-diabetic European Americans with fasting insulin $(\mathrm{OR}=1.84, \mathrm{P}=$ $0.210)$ was stronger at the highest insulin quartile $(O R=4.08, P=0.040)$.
\end{abstract}

Conclusions: As demonstrated with type 1 diabetic nephropathy, the GLUT1 Enh2 risk genotype, instead of Xbal, may be associated with type 2 diabetic albuminuria among European Americans, though an association is not conclusive. The association among diabetic European Americans found in stage 1 was not replicated in stage 2; however, this risk association was evident after combining all diabetic European Americans from both stages. Additionally, our results suggest this association may extend to non-diabetics with high insulin concentrations. Rarity of the Enh2 risk genotype among African Americans precludes any definitive conclusions, although data suggest a risk-enhancing role.

\footnotetext{
* Correspondence: coresh@jhu.edu

'Departments of Medicine and Epidemiology, Johns Hopkins Medical

Institutions, Baltimore, Maryland, USA

Full list of author information is available at the end of the article
} 


\section{Background}

Glucose transporter 1(GLUT1) is the major facilitative glucose transporter in glomerular mesangial cells [1]. Studies show glucose transport as the rate limiting step in mesangial expansion, and alterations of GLUT1 activity may stimulate extracellular matrix(ECM) production [2] even under normoglycemic conditions [3]. Additionally, experimental evidence suggests GLUT1 may be associated with hypertensive glomerulopathy [4].

However, results of case-control studies of GLUT1 sequenece variations (the $\mathrm{XbaI}$ polymorphism, a C-to-A transversion in intron 2: see figure 1) and diabetic nephropathy have been inconsistent [5-11] with significant heterogeneity between studies [12]. Most studies demonstrated risk transmitted in a recessive fashion $[5,6,8,11,13]$. Few studies examined diabetic nephropathy in relation to GLUT1 SNPs other than XbaI $[5,11,13]$. A study of those with type 1 diabetes examined six GLUT1 single nucleotide polymorphisms(SNPs) and found homozygosity for the XbaI A allele was associated with diabetic nephropathy [5]. Additionally, putative human enhancer elements were identified [5], including the insulin-responsive enhancer-2(see figure 1), and homozygosity for the minor allele(C-to-T) of the enhancer-2 SNP1(Enh2 SNP) was also associated with type 1 diabetic nephropathy [5]. Though Enh2 SNP has strong linkage disequilibrium with $\mathrm{XbaI} S \mathrm{SP}$, more evidence suggests Enh2 SNP may be causally related to diabetic nephropathy [5]. More recently, a study extended the significant association of the Enh2 SNP risk genotype to those with type 2 diabetic nephropathy; however, a statistically significant association between $\mathrm{XbaI}$ genotypes and type 2 diabetic nephropathy was not demonstrated [11].

There has been no investigation among non-diabetics or African Americans of albuminuria and GLUT1 genetic variation, especially for the Enh2 SNP. Additionally, the Enh2 SNP is not readily available on most candidate gene chips, with available proxy SNPs having low $r^{2}$. We conducted a case-control study nested within a large biracial community-based middle-aged cohort to determine the risk of albuminuria associated with six

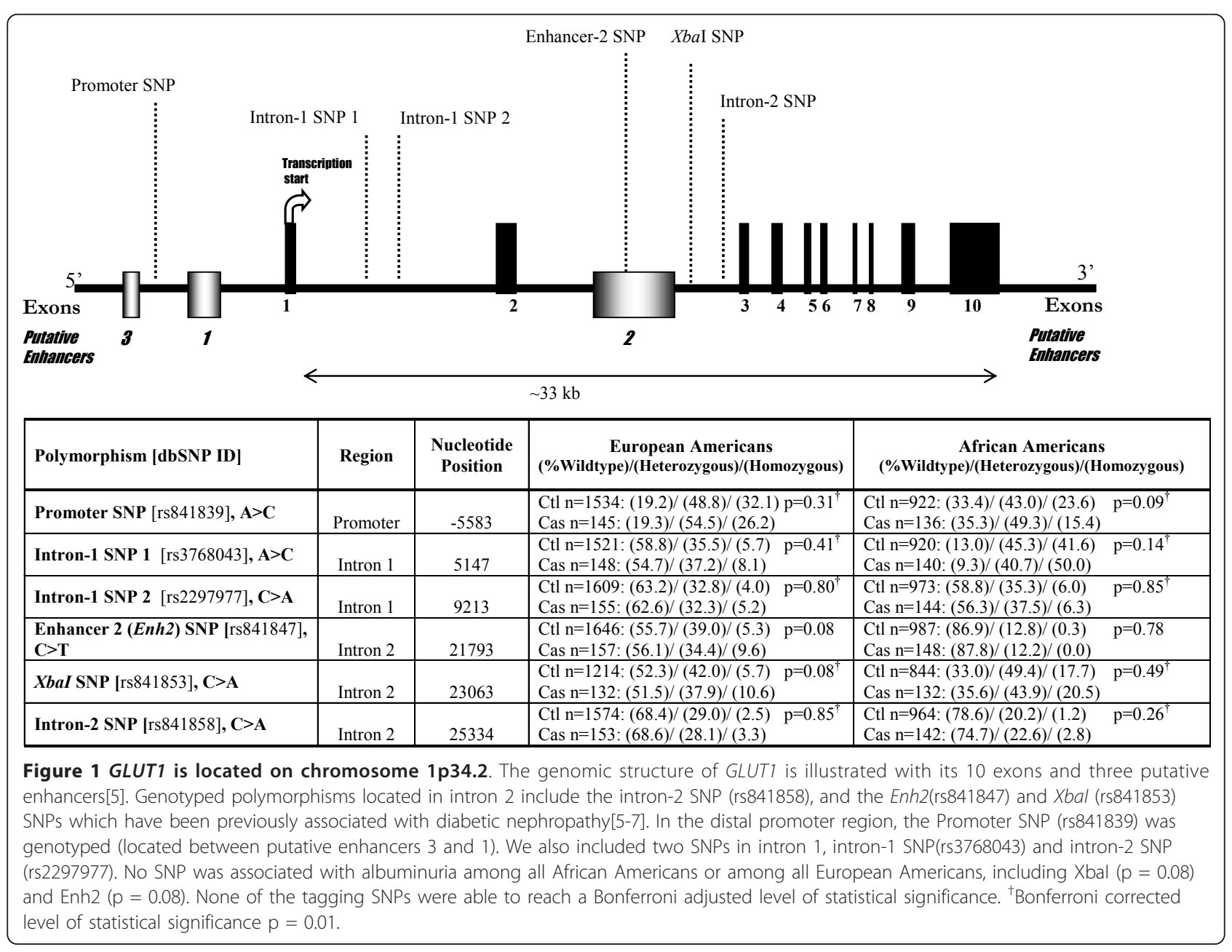


GLUT1 SNPs by race and by type 2 diabetes status. We performed a two-stage analysis in our population( $\mathrm{n}=$ 10,278): 1) an initial evaluation of all six candidate GLUT1 SNPs among stage 1 participants $(\mathrm{n}=2938)$, and 2) a subsequent phase (stage $2, \mathrm{n}=7340$ ) to assess the likeliest stage 1 candidate SNP. Because enhancer- 2 is a putative insulin-responsive element [5], we hypothesized insulin may interact with the Enh2 SNP to affect risk of albuminuria.

\section{Methods}

\section{Study Design and Population}

The study population consisted of African Americans and European Americans with and without type 2 diabetes in the Atheroslcerosis Risk in Communities (ARIC) Study, aged 45 to 64 years at baseline in 1987 through 1989, from 4 US communities: Forsyth County, NC; Jackson, MS; suburbs of Minneapolis, MN, and Washington Co., MD. Participants underwent four standardized examinations in field center clinics, scheduled approximately every three years [14] with approval of the institutional review boards.

Of the 11,625 African American and European American participants who attended visit four, urinary albumin and creatinine measurements were available for 11,447 participants. All measurements were from ARIC visit 4 , as urine samples were only collected at this visit. We excluded those missing visit 4 serum creatinine $(\mathrm{n}=$ 182), information on diabetes, hypertension, systolic blood pressure (SBP), diastolic blood pressure (DBP), or body mass index (BMI) $(n=125)$, and those without available DNA ( $\mathrm{n}=69$ ). For stage 1 , we genotyped six GLUT1 SNPs (including Enh2 and XbaI) in a sample of the above participants (sample $1, \mathrm{n}=3,204$ ), enriched for African Americans and people with type 2 diabetes, a convenience sample previously used for the study of genetic risk factors in cardiovascular disease and type 2 diabetes. Sample 1 was an otherwise unselected portion of the total population. We genotyped only the most likely candidate SNP (Enh2) from stage 1 in the remaining participants who met our inclusion criteria from ARIC visit 4, individuals who comprised stage 2 (sample 2 , $\mathrm{n}=7,867)$. Missing genotypes were excluded from stages $1(\mathrm{n}=266)$ and $2(\mathrm{n}=527)$. With exclusion criteria, of the total study population ( $n=10,278)$, sample $1(\mathrm{n}=2938)$ was $28.6 \%$ and sample $2(\mathrm{n}=7340)$ was 71.4\%. Participants from samples 1 and 2 were mutually exclusive.

Of those who attended visit 4, the 1,347 excluded individuals were older (63.1 vs. 62.8 years of age), more likely to be African American (37.7\% vs. 21.0\%), type 2 diabetic (22.5\% vs. $16.2 \%)$, hypertensive (57.2\% vs. $46.7 \%)$, and male $(46.5 \%$ vs. $43.8 \%)$ compared to included participants.

\section{Assessment of Clinical Characteristics}

Clinical charcteristics were assessed during visit 4 . A standardized interview, clinical examination, and laboratory investigation collected demographic, anthropometric, and cardiovascular risk factor data for participants [15]. Fasting blood sample collection and processing is described elsewhere [15]. Two standardized blood pressure measurements were performed by trained technicians, and their average was used. Hypertension was defined as a systolic blood pressure (SBP) $\geq 140 \mathrm{mmHg}$, a diastolic blood pressure (DBP) $\geq 90$ $\mathrm{mmHg}$, or the self-reported use of antihypertensive medication during the previous 2 weeks. Diabetes mellitus was defined as fasting glucose $\geq 126 \mathrm{mg} / \mathrm{dL}$, nonfasting glucose $\geq 200 \mathrm{mg} / \mathrm{dL}$, or self-reported history or treatment of type 2 diabetes. Visit 4 glomerular filtration rate (GFR) was estimated from calibrated serum creatinine using the simplified equation developed using data from the Modification of Diet in Renal Disease (MDRD) Study $[16,17]$ as follows:

GFR $\mathrm{mL} / \mathrm{min} / 1.73 \mathrm{~m}^{2}=186.3 *(\mathrm{SCr})^{-1.154 *(\text { age })^{-0.203}}$ * (0.742 if female $) *(1.21$ if African American $)$

\section{Ascertainment of Albuminuria}

An untimed urine sample was collected during visit 4. Aliquots were frozen within 12 hours and stored at $-70^{\circ} \mathrm{C}$. Albumin and creatinine concentrations were measured in the University of Minnesota Physicians Outreach Laboratories, Minneapolis, Minnesota, with albumin by a nephelometric method on the Dade Behring BN100 (assay sensitivity, $2.0 \mathrm{mg} / \mathrm{L}$ ), and creatinine using the Jaffe method on a Beckman CX3 to determine albumin-to-creatinine ratios (ACR; ug/mg) for participants. Blinded samples $(n=516)$ analyzed for quality assurance showed a correlation coefficient $\left.{ }^{(}\right)$of the $\log _{e^{-}}$ transformed ACR as $r=0.95$. ACR was used to determine levels of albuminuria according to American Diabetes Association [18] and National Kidney Foundation [19] recommendations: normoalbuminuria (ACR < $30 \mu \mathrm{g} / \mathrm{mg}$ ), microalbuminuria (ACR 30-299 $\mu \mathrm{g} / \mathrm{mg}$ ), and macroalbuminuria (ACR $\geq 300 \mu \mathrm{g} / \mathrm{mg}$ ). Normoalbuminuric participants were classified as controls. Albuminuria cases had either microalbuminuria or macroalbuminuria. Of the total study, there were 825 cases of albuminuria, $37.0 \%(\mathrm{n}=305)$ in sample 1 and $63.0 \%(\mathrm{n}=520)$ in sample 2 . For sensitivity analysis, microalbuminuria and macroalbuminuria cases were examined separately, compared to controls.

\section{GLUT1 Candidate SNP Selection and Genotyping}

GLUT1 consists of 10 exons including three recently described putative human enhancers (see Figure 1) [5] with murine and rat homology $[20,21]$. The XbaI SNP [5-7] and the Enh2 SNP [5,11] were chosen as 
candidates for genotyping based on previous studies. Using the human GLUT1 genomic sequence(GenBank accession no. NT_032977) and the public SNP database dbSNP build 120, it was determined the XbaI SNP corresponds to rs 841853 and the Enh2 SNP corresponds to rs841847. The dbSNP rs841839 C > A transversion (denoted Promoter SNP) was chosen for genotyping based on its proximity to both enhancers-3 and -1 . At the time of genotyping, the Hap Map Phase II data were not available; therefore, 3 additional SNPs were chosen with an average distance between SNPs of $4.2 \mathrm{~kb}$ to provide coverage of the gene $(76.8 \%)$. Three additional SNPs selected were: intron-1 SNP1(rs3768043), intron-1 SNP2(rs2297977), and intron-2 SNP(rs841858).

For the evaluation phase, genotypes in sample 1 were assessed by PCR amplification of genomic DNA and completed for rs841847(Enh2 SNP) using Pyrosequencing technology (Pyrosequencing, Uppsala, Sweden) as previously described [22]. For the SNPs rs841853 (XbaI), rs841839, rs3768043, rs2297977, and rs841858, genotypes were completed using Orchid SNPstream UHT genotyping system (Orchid Bioscience) as previously described [23]. For sample 1, the error rate on the basis of blind replicates $(\mathrm{n}=444)$ for the Orchid SNPstream UHT genotyping system was $0.2 \%$. For the replication phase, genotypes for rs841847 in sample 2 were assessed and completed using Pyrosequencing technology.

\section{Statistical Analysis}

For stage $1(\mathrm{n}=2938)$, genotype frequencies of all SNPs were tested for consistency with Hardy-Weinberg expectations by the $\chi^{2}$ test within race strata among controls consisting of non-diabetic individuals without albuminuria. Because allele frequencies differed by race, analyses were race-stratified. Tests of differences in clinical characteristics by case status included t-tests, ANOVA, and $\chi^{2}$ tests. Logistic regression models calculated the odds ratio (OR) and 95\% confidence interval (95\% CI) of albuminuria for each SNP, using either genotypic or recessive models for inheritance. Our genotypic associations of XbaI and Enh2 in European Americans suggest recessive modes of action, similar to previous findings $[5,6]$. To differentiate the effects of the likely candidate from remaining GLUT1 SNPs, multivariate logistic regression was performed, adjusting for type 2 diabetes, XbaI, Enh2, rs841839, rs841858, rs2297977, and rs3768043 genotypes.

There was strong prior evidence for Enh2 (rs841847) as a functional candidate polymorphism, both in human and laboratory studies [5,11,24-29]. Based on this a priori determination of the status of Enh2 (rs841847) as a functional candidate SNP, we believed it was appropriate to keep the alpha for statistical significance of $p<$ 0.05 for analyses focusing on Enh2 (rs841847) with further correction. However, given that the other 5 SNPs (rs841853, rs841839, rs3768043, rs2297977, rs841858) examined were largely meant to be tagging SNPs without any prior evidence to suggest function, we felt it necessary to correct for multiple testing errors by applying a Bonferroni correction [30], with the corrected alpha for statistical significance at the 0.05 level determined by dividing the significance level by the number of tagging SNPs, with adjusted p-value $<0.01$.

Using the Bayesian method as implemented in PHASE v2.1 [31,32], haplotypes were inferred for all individuals with genotype data for at least five of the six SNPs (rs841847, rs841853, rs841839, rs3768043, rs2297977, rs841858) separately for African Americans $(\mathrm{n}=1,036)$ and European Americans $(\mathrm{n}=1638)$. Phase-formatted data were run as race-specific files which combined case and control subjects, a more conservative estimation of haplotype frequency than separate case and control sample analyses. Pairwise SNP linkage disequilibrium (LD) was measured with D' and $\mathrm{r}^{2}$ [33] in HaploView (Cambridge, MA). Categorized by major haplotypes (> $5 \%$ frequency), haplotype frequency was compared between case and control groups with $\chi^{2}$ test. For haplotype analyses, a Bonferroni correction was also applied by dividing the significance level $(0.05)$ by the number of major haplotypes ( $\mathrm{n}=4$ for European Americans, $\mathrm{n}=$ 8 for African Americans) for haplotype-based association analysis (Bonferroni corrected level of statistical significance for European Americans $\mathrm{p}<0.0125$, for African Americans p < 0.00625). Associations with albuminuria for each diplotype (of major haplotypes) were examined separately using logistic regression. For diplotype analyses for European Americans, a Bonferroni correction was also applied by dividing the significance level by the number of diplotypes $(n=10)$ comprised of the major haplotypes, with adjusted level of statistical significance $\mathrm{p}<0.005$.

In stage $2(\mathrm{n}=7340)$ the Enh2 SNP was genotyped. Associations of albuminuria/microabluminuria/macroalbuminuria and the Enh2 risk genotype were assessed using multivariate logistic regression models for the total study population, stratified by race and type 2 diabetes. Covariates included age, gender, systolic blood pressure, diastolic blood pressure, hypertension medication use, BMI, estimated GFR, and fasting glucose. Additionally, among non-diabetics, we examined interactions of albuminuria/macroalbuminuria for the Enh2 risk genotype with quartiles of fasting insulin, based on the Enh2 SNP location in the putative insulin-responsive enhancer 2 [5]. Multivariate linear regression was used to assess associations between the Enh2 risk genotype with log-transformed ACR $\mu \mathrm{g} / \mathrm{mg}$ (data log-transformed due to the skewed distribution) and also serum creatinine among European Americans, stratified by type 2 
diabetes; these analyses were not performed among African Americans due to the rarity of the Enh2 risk genotype in that population.

\section{Results \\ GLUT1 SNPs}

Six SNPs (average spacing $4.2 \mathrm{~kb}$ ) of the GLUT1 genomic region (Promoter SNP, Intron-1 SNPs 1 and 2, Enhancer 2 SNP, XbaI SNP, and Intron 2 SNP) were genotyped in an unrelated sample of 1803 European Americans and 1135 African Americans in stage 1 (Figure 1). In European Americans, all SNPs were in Hardy-Weinberg Equilibrium (HWE). Among African Americans, the Promoter SNP was not in HWE $(\mathrm{P}<$ 0.05). XbaI minor allele(A) frequency in African Americans was higher than in European Americans (frequency = 0.42 versus 0.27). In European Americans, the XbaI A allele and the Enh2 T allele (frequency $=0.25$ ) were comparable to previously published estimates $[5,6,8,9,11]$. However, minor allele of Enh2(T) was low in African Americans (frequency $=0.07$ ).

The five SNPs (Intron-1 SNPs 1 and 2, Enh2 SNP, XbaI SNP, and Intron-2 SNP) downstream of Promoter SNP were in strong LD with each other (range of D' = 0.95 to 1.00 in European Americans and 0.96 to 1.00 in African Americans) [34]. The promoter SNP was separated from Intron-1 SNP1 by $\sim 10 \mathrm{~kb}$ and was in greater LD among European Americans (D' = 0.69) than African Americans (D' = 0.09). Among both African Americans and European Americans, Enh2 and XbaI SNPs were in strong LD ( $\left.D^{\prime}=0.96\right)$; however, the $r^{2}$ was much lower in African Americans compared to European Americans (0.09 vs. 0.70) because frequencies of Enh2 and XbaI significantly differed in African Americans.

\section{Clinical Characteristics}

In both race groups in stage 1 , the case subjects had worse risk factor profiles than controls (Table 1). Risk factor profiles were also worse for case subjects among participants from stage $2(n=7340)$.

\section{Stage 1: Evaluation Phase of Six GLUT1 SNP Genotypes by Albuminuria Case Status European Americans}

Among European Americans in stage 1, GLUT1 SNP genotype distributions were not significantly different by case status, though the Enh2 SNP approached statistical significance $(\mathrm{P}=0.08$, figure 1$)$. $\mathrm{XbaI}$ and other tagging SNPs did not approach the Bonferroni corrected level of statistical significance of $\mathrm{p}<0.01$. For Enh2 T allele, heterozygotes had an $\mathrm{OR}=0.88$, while homozygotes had OR = 1.77 (95\% CI: $0.98-3.20)$. Similarly for the XbaI A allele, heterozygotes had OR 0.92 while homozygotes had OR 1.89 (95\% CI: 1.01 - 3.55). Our genotypic associations of $\mathrm{XbaI}$ and Enh2 in European Americans suggest a recessive model, similar to previous findings $[5,6,11]$.

Albuminuria was associated with the Enh2 TT risk genotype with OR 2.36 (95\% CI: $1.09-5.09, \mathrm{P}=0.025)$ compared to those without the risk genotype (CC or CT) among those with type 2 diabetes. This association was not present among non-diabetics (OR 1.31, P = 0.574 , Table 2). Similarly, the XbaI risk genotype (AA) had a larger association with albuminuria among those with type 2 diabetes rather than non-diabetics (OR 2.23 $[\mathrm{P}=0.064]$ and $1.75[\mathrm{P}=0.212]$, respectively), though associations were not statistically significant for $\mathrm{XbaI}$ and did not approach the Bonferroni corrected level of statistical significance $(\mathrm{p}<0.01)$ (Table 2). Among European Americans stratified by diabetes, besides Enh2 no other GLUT1 polymorphisms were associated with albuminuria (results not shown). In all European Americans, multivariate analysis adjusting for diabetes simultaneously examined the association with albuminuria of all six GLUT1 SNPs, and the Enh2 risk genotype had an increased OR with albuminuria $(\mathrm{OR}=3.37,95 \% \mathrm{CI}$ : $0.83-13.78, \mathrm{p}=0.090)$ whereas $\mathrm{XbaI}$ did not $(\mathrm{OR}=$ 0.94 , 95\% CI: $0.26-3.42, \mathrm{p}=0.931$ ). For the four remaining SNPs, genotypic ORs ranged from 0.72 to 1.85 (all p-values > 0.17) with none of the SNPs approaching the Bonferroni adjusted statistical significance level of $\mathrm{p}<0.01$.

Similarly, detailed haplotype and diplotype analyses supported a potential role of the Enh2 TT genotype in raising the risk of albuminuria in a recessive fashion (Table 3). When the ten diplotypes associated with the major haplotypes A, B, C, and D (table 3) were examined among European Americans, only the BD diplotype was associated with albuminuria (OR 3.03, 95\% CI 1.36 - 6.79, $\mathrm{p}=0.007$, compared to individuals without the $\mathrm{BD}$ diplotype). However, this approached but did not reach a Bonferroni corrected level of statistical significance of $\mathrm{p}<0.005$. Additionally, the diplotypes consisting of the B or D "risk" haplotypes were rare among European Americans, with BB, BD, and DD diplotypes accounting for $2.7 \%, 2.2 \%$, and $0.5 \%$ of the 1663 European Americans with available diplotypes analyzed. Overall, haplotype diplotypes homozygous for the Enh2 $\mathrm{T}$ allele had an increased risk of albuminuria compared to all others combined $(\mathrm{P}=0.014)$, though it did not reach the Bonferroni corrected level of statistical significance of $\mathrm{p}<0.005$.

\section{African Americans}

Among African Americans, no GLUT1 SNP was significantly associated with case status. Frequency of the Promoter SNP genotype was slightly but not significantly different by case status (see figure 1). Enh2 TT genotype was rare $(0.3 \%$ in controls, $0.0 \%$ in cases $)$ and did not 
Table 1 Baseline Characteristics. Clinical characteristics of study subjects by Race and Albuminuric Status

\begin{tabular}{|c|c|c|c|c|c|c|c|}
\hline \multirow[b]{3}{*}{ Characteristic } & \multicolumn{3}{|c|}{ Stage 1} & \multicolumn{4}{|c|}{ Stage 2} \\
\hline & \multicolumn{3}{|c|}{ European Americans $(n=1803)$} & \multicolumn{4}{|c|}{ European Americans $(n=6319)$} \\
\hline & Control Subjects & Albur & minuria Cases & Control & Subjects & Albu & minuria Cases \\
\hline $\mathbf{N}$ & 1646 & & 157 & & 945 & & 374 \\
\hline Age, mean(SD), y & $63.2(5.6)$ & 64.9 & $(5.6)^{* * *}$ & 62.8 & $(5.6)$ & 65.2 & $(5.6)^{* * *}$ \\
\hline Male, No. (\%) & $802(48.7)$ & 92 & $(58.6)^{*}$ & 2655 & $(44.7)$ & 199 & $(53.2)^{* *}$ \\
\hline Hypertension, No. (\%) & 731 (44.4) & 111 & $(70.7)^{* * *}$ & 2266 & $(38.1)$ & 269 & $(71.9)^{* * *}$ \\
\hline Type 2 Diabetes, No. (\%) & $480 \quad(29.2)$ & 84 & $(53.5)^{* * *}$ & 428 & $(7.2)$ & 103 & $(27.5)^{* * *}$ \\
\hline Body mass index, $\mathrm{kg} / \mathrm{m}^{2}$ & $29.0 \quad(5.5)$ & 30.0 & $(6.3)^{*}$ & 28.0 & $(5.0)$ & 28.8 & $(6.0)^{* *}$ \\
\hline SBP $(\mathrm{mmHg})$ & $126.8(17.4)$ & 135.7 & $(20.4)^{* * *}$ & 124.4 & $(17.8)$ & 138.3 & $(23.2)^{* * *}$ \\
\hline $\mathrm{DBP}(\mathrm{mmgHg})$ & $69.7(10.0)$ & 70.0 & $(11.1)$ & 69.5 & $(9.6)$ & 72.5 & $(12.1)^{* * *}$ \\
\hline Fasting Glucose $(\mathrm{mg} / \mathrm{dL})^{\S}$ & $115.4(34.9)$ & 134.4 & $(52.2)^{* * *}$ & 102.1 & $(21.2)$ & 116.8 & $(43.4)^{* * *}$ \\
\hline GFR, $\mathrm{ml} / \mathrm{min}$ per $1.73 \mathrm{~m} 2$ & $81.7 \quad(16.9)$ & 75.0 & $(25.7)^{* * *}$ & 80.7 & $(16.1)$ & 76.1 & $(22.3)^{* * *}$ \\
\hline \multirow[t]{2}{*}{ ACR, $\mu \mathrm{g} / \mathrm{mg}$, median (25\%ile, $75 \%$ ile) } & $3.7 \quad(1.8,6.7)$ & 102.6 & $(47.4,275.0)^{* * *}$ & 3.6 & $(2.0,6.5)$ & 74.8 & $(44.4,166.7)^{* * *}$ \\
\hline & \multicolumn{3}{|c|}{ African Americans $(n=1135)$} & \multicolumn{4}{|c|}{ African Americans $(n=1021)$} \\
\hline Characteristic & Control Subjects & \multicolumn{2}{|c|}{ Albuminuria Cases } & \multicolumn{2}{|c|}{ Control Subjects } & \multicolumn{2}{|c|}{ Albuminuria Cases } \\
\hline $\mathbf{N}$ & 987 & \multicolumn{2}{|c|}{148} & \multicolumn{2}{|c|}{875} & \multicolumn{2}{|c|}{146} \\
\hline Age, mean(SD), y & $61.3(5.5)$ & 63.0 & $(5.8)^{++\dagger}$ & 61.8 & $(5.7)$ & 62.5 & $(5.7)$ \\
\hline Male, No. (\%) & $336(34.0)$ & 44 & $(29.7)$ & 324 & $(37.0)$ & 54 & $(37.0)$ \\
\hline Hypertension, No. (\%) & $615(62.3)$ & 127 & $(85.8)^{\dagger+\dagger}$ & 558 & $(63.8)$ & 127 & $(87.0)^{\mathrm{t+ \dagger}}$ \\
\hline Type 2 Diabetes, No. (\%) & $217(22.0)$ & 80 & $(54.1)^{\dagger+\dagger}$ & 196 & $(22.4)$ & 80 & $(54.8)^{\mathrm{t+ \dagger}}$ \\
\hline Body mass index, $\mathrm{kg} / \mathrm{m}^{2}$ & $30.7(6.5)$ & 30.8 & $(6.6)$ & 30.3 & $(5.9)$ & 31.4 & $(6.6)^{\dagger}$ \\
\hline SBP $(\mathrm{mmHg})$ & $131.2(18.4)$ & 144.4 & $(23.2)^{\dagger+\dagger}$ & 132.0 & $(18.9)$ & 144.8 & $(22.5)^{\mathrm{t+ \dagger}}$ \\
\hline DBP (mmgHg) & $75.0(10.0)$ & 78.2 & $(13.0)^{t+\dagger}$ & 75.7 & $(10.1)$ & 78.5 & $(11.9)^{t+}$ \\
\hline Fasting Glucose $(\mathrm{mg} / \mathrm{dL}){ }^{\S}$ & 114.3 (39.6) & 136.4 & $(62.2)^{++\dagger}$ & 112.7 & $(38.5)$ & 137.9 & $(62.8)^{t+\dagger}$ \\
\hline GFR, $\mathrm{ml} / \mathrm{min}$ per $1.73 \mathrm{~m} 2$ & $91.5(19.0)$ & 83.0 & $(29.8)^{t+\dagger}$ & 90.9 & $(21.0)$ & 80.7 & $(29.3)^{t+\dagger}$ \\
\hline ACR, $\mu \mathrm{g} / \mathrm{mg}$, median ( $25 \%$ ile, $75 \%$ ile) & $1.9(0.7,4.7)$ & 95.9 & $(47.2,363.2)^{\dagger+\dagger}$ & 2.2 & $(0.8,5.2)$ & 81.7 & $(44.7,249.7)^{++}$ \\
\hline
\end{tabular}

Data are means (SD), number (\%), or for ACR median (25\%ile, 75\%ile). European Americans: ${ }^{*} \mathrm{P}<0.05$; ${ }^{*} \mathrm{P}<0.01$; ${ }^{* * *}<0.001$.

African Americans: $\mathrm{tP}<0.05 ; \mathrm{t} \mathrm{P}<0.01 ;+\mathrm{t} \mathrm{P}<0.001$.

${ }^{\S}$ Individuals with fasting glucose measured. Evaluation set (European Americans $n=1582$ controls and $n=150$ cases; African Americans $n=922$ controls and $n$ $=113$ cases). Valdiation set (European Americans $n=5822$ controls and $n=356$ cases; African Americans $n=787$ controls and $n=113$ cases).

allow examination with case status. There was sufficient power to examine the XbaI SNP (with the minor A allele frequency of $42.4 \%$ ) and case status in African Americans, which was not $\operatorname{significant}(\mathrm{P}=0.5)$. By diabetes status, the XbaI AA risk genotype was not associated with albuminuria either among those with type 2 diabetes (OR 1.05, 95\% CI: 0.53 - 2.09) or without diabetes (OR 1.34, 95\% CI: 0.70 - 2.56). Further detailed haplotype and diplotype analyses did not demonstrate any significant associations with albuminuria among African Americans ( $\mathrm{P}=0.877$, table 3$)$.

\section{GLUT1 Enh2 and Albuminuria in Samples from Stages 1 and 2}

Based on stage 1, the Enh2 SNP appeared to be the risk polymorphism affecting albuminuria, particularly among European Americans with type 2 diabetes. The GLUT1 Enh2 polymorphism was genotyped in a separate stage 2 of European Americans ( $\mathrm{n}=6319 ; 374$ cases $)$ and
African Americans ( $\mathrm{n}=1021,146$ cases), and the risk genotype tended towards an increased (but not statistically significant) OR among European Americans with diabetes $(\mathrm{OR}=1.66,95 \% \mathrm{CI}: 0.77-3.57, \mathrm{p}=0.192)$ but not among non-diabetics (OR $=0.99,95 \% \mathrm{CI}$ : 0.61 1.59, $\mathrm{p}=0.953)$. The risk association among European Americans with diabetes from stage 1 was not replicated in stage 2. In the stage 2 sample of African Americans, the GLUT1 Enh2 risk genotype was still rare $(0.1 \%$ of controls and $1.4 \%$ of cases, Chi-square(2d.f.) $\mathrm{P}=0.03$ ) precluding informative analysis by diabetes status.

By race, we combined the samples from stages 1 and 2 for European Americans $(\mathrm{n}=8,122)$ and African Americans $(\mathrm{n}=2156)$ and examined risk-factor adjusted associations with albuminuria and the GLUT1 Enh2 risk genotype by diabetes status (Table 4). Among European Americans with diabetes, the Enh2 risk genotype had an increased risk-factor adjusted OR with albuminuria (OR 2.11, $\mathrm{p}=0.010$ ), microalbuminuria (OR 2.01, $\mathrm{p}=0.034$ ) 
Table 2 Stage 1GLUT1 Enh2 and Xbal Risk genotype distributions among case and control subjects and odds ratio of albuminuria, by diabetes and race

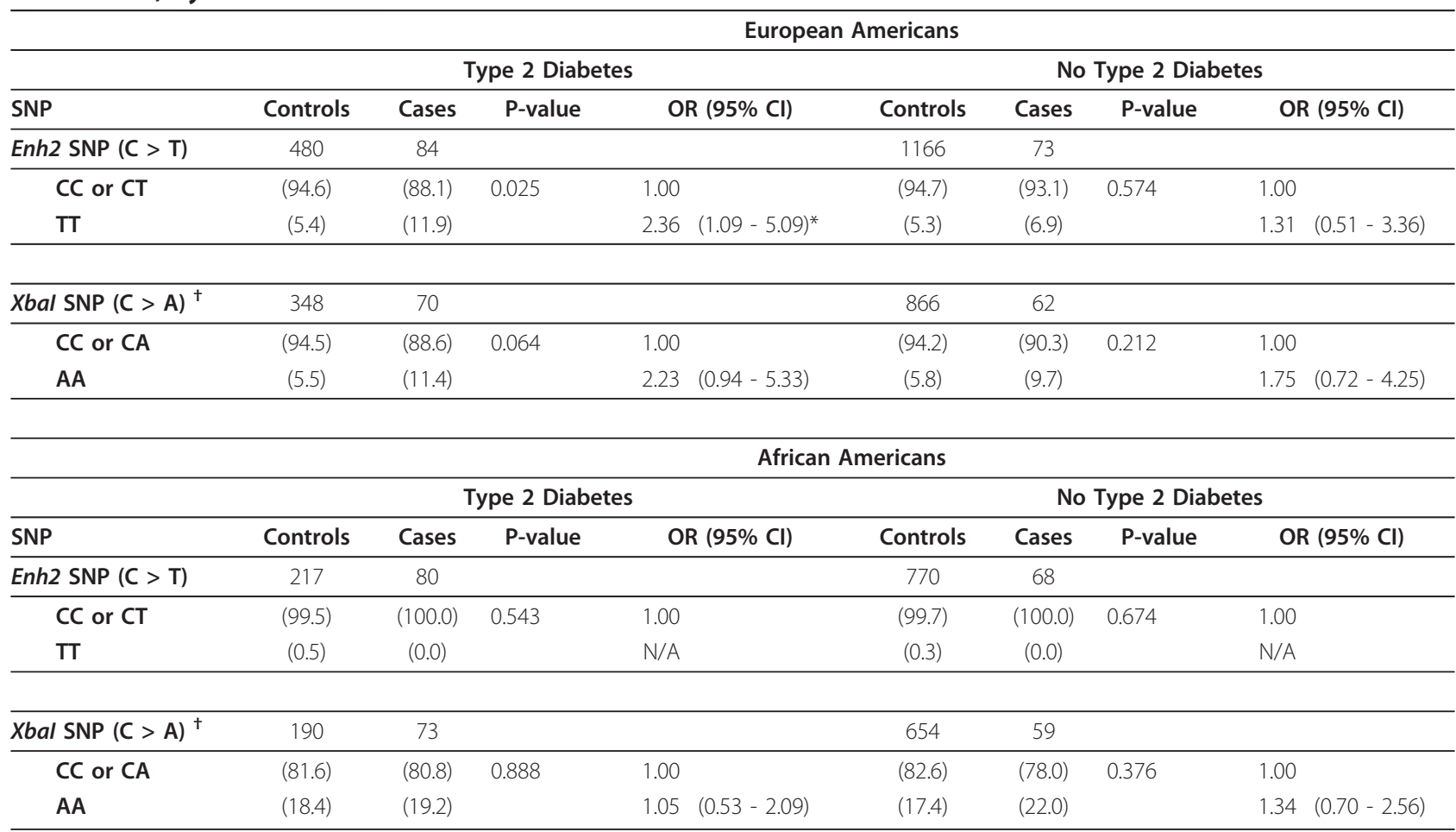

*P $<0.05$.

${ }^{\dagger}$ Bonferroni correction requires $\mathrm{p}<0.01$ to reach statistical significance.

Table 3 Major Haplotype Distribution in Case and Control Subjects by Race in stage $\mathbf{1}^{\text {t\# }}$

\begin{tabular}{|c|c|c|c|c|c|c|c|c|c|c|}
\hline $\begin{array}{l}\text { European } \\
\text { Americans }\end{array}$ & Haplotype & $\begin{array}{l}\text { Promoter } \\
\text { SNP }\end{array}$ & $\begin{array}{l}\text { Intron-1 } \\
\text { SNP1 }\end{array}$ & $\begin{array}{l}\text { Intron-1 } \\
\text { SNP2 }\end{array}$ & $\begin{array}{l}\text { Enh2 } \\
\text { SNP }\end{array}$ & $\begin{array}{l}\text { Xbal } \\
\text { SNP }\end{array}$ & $\begin{array}{l}\text { Intron-2 } \\
\text { SNP }\end{array}$ & Controls & $\begin{array}{r}\text { Albuminuria } \\
\text { Cases } \\
\end{array}$ & $\begin{array}{r}\mathrm{P}- \\
\text { value } \\
\end{array}$ \\
\hline \multirow{5}{*}{$\begin{array}{l}\mathrm{N}=3326 \\
\text { Haplotypes }\end{array}$} & A & C & A & C & C & C & C & $(43.4)$ & $(39.5)$ & 0.773 \\
\hline & B & A & A & C & $\mathrm{T}$ & A & C & $(17.2)$ & $(18.9)$ & \\
\hline & $\mathrm{C}$ & A & C & A & C & C & A & $(14.1)$ & $(14.5)$ & \\
\hline & D & C & A & C & $\mathrm{T}$ & A & C & $(6.2)$ & $(6.8)$ & \\
\hline & Others & * & * & * & * & * & * & $(19.1)$ & $(20.3)$ & \\
\hline \multicolumn{11}{|c|}{ African Americans } \\
\hline \multirow{9}{*}{$\begin{array}{l}\mathrm{N}=2156 \\
\text { Haplotypes }\end{array}$} & A & C & A & C & C & C & C & (13.7) & $(10.7)$ & 0.877 \\
\hline & B & A & A & C & $\mathrm{T}$ & A & C & $(5.4)$ & $(5.0)$ & \\
\hline & $\mathrm{C}$ & A & C & A & C & C & A & $(7.7)$ & (9.6) & \\
\hline & $E$ & C & $C$ & C & C & C & C & (14.0) & (13.9) & \\
\hline & $F$ & C & C & C & C & A & C & (11.0) & (12.1) & \\
\hline & G & A & C & C & C & A & C & (12.7) & (11.4) & \\
\hline & $\mathrm{H}$ & A & C & A & C & C & C & (11.9) & (12.1) & \\
\hline & I & A & A & C & C & A & C & $(6.8)$ & (7.5) & \\
\hline & Others & * & $*$ & * & * & * & * & (16.8) & (17.5) & \\
\hline
\end{tabular}

₹ Major haplotypes have a frequency $\geq 5 \%$. Minor alleles are shaded in gray. Bonferroni corrected level of statistical significance for European Americans $p<$ 0.0125 and for African Americans $\mathrm{p}<0.00625$.

${ }^{\dagger}$ Among European Americans, the ten diplotypes associated with the major haplotypes A, B, C, and D from table 3, only the BD diplotype was associated with albuminuria (OR 3.03, 95\% Cl $1.36-6.79, p=0.007$, compared to individuals without the BD diplotype). However, this did not reach a Bonferroni corrected level of statistical significance of $p<0.005$. The diplotypes consisting of the B or D "risk" haplotypes were rare, with BB, BD, and DD diplotypes accounting for $2.7 \%$, $2.2 \%$, and $0.5 \%$ of the 1663 European Americans with available diplotypes analyzed. 
and macroalbuminuria (OR 2.69, $\mathrm{p}=0.045)$ (Model 1, Table 4). Further adjustment for BMI and estimated renal function (model 2) did not significantly affect the associations (with albuminuria OR 2.14, $\mathrm{p}=0.009$ ), nor did further adjustment for fasting glucose (OR 1.99, p = 0.029). However, among European Americans without type 2 diabetes, Enh2 was not associated with albuminuria (OR 1.01, $\mathrm{p}=0.980$ ) and was moderately but not significantly associated with macroalbuminuria (OR 1.64, 95\% CI: 0.64-4.25, $\mathrm{p}=0.305$ ) (Model 1, Table 4). Additional adjustment for visit 2 hemoglobin A1C (HbA1C) in a sub-sample did not significantly change point estimates with albuminuria $(\mathrm{OR}=1.87,95 \% \mathrm{CI}$ : $1.02-3.42$ in diabetic individuals, $\mathrm{OR}=1.25,95 \% \mathrm{CI}$ : $0.63-2.46$ in non-diabetics). There was no evidence of interaction with hypertension (results not shown). Additionally, among European Americans, we also examined potential associations using multivariate linear regression with log-transformed ACR $\mu \mathrm{g} / \mathrm{mg}$ (data logtransformed due to the skewed distribution) and also serum creatinine among European Americans, stratified by type 2 diabetes (see Table 5). Though the Enh2 risk genotype tended to be positively associated with worse ACR and higher serum creatinine only among type 2 diabetic European Americans, there were no statistically significant associations. We did not perform this analysis among African Americans given the low frequency of the Enh2 risk genotype among this population.

Among African Americans, after adjustment for model 2 covariates, the GLUT1 Enh2 risk genotype had a positive association with albuminuria and microalbuminuria among those with type 2 diabetes $(\mathrm{OR}=4.36$ and 6.39 with $\mathrm{p}=0.321$ and 0.203 , respectively) and without diabetes $(\mathrm{OR}=4.69$ and 5.15 with $\mathrm{p}=0.187$ and 0.164 , respectively), though it was not significant due to low frequency of the SNP.

Table 4 Adjusted relative odds of albuminuria, microalbuminuria, and macroalbuminuria for Enh 2 in all genotyped European Americans and African Americans, by type 2 diabetes status GLUT1 Enh2 Risk Genotype(TT) Relative Odds (95\% Confidence Interval) reference: (CC or CT)

\begin{tabular}{|c|c|c|c|c|c|c|}
\hline \multirow{2}{*}{$\begin{array}{l}\text { Model }^{\dagger} \text { (N = Enh2 TT Genotype/All Genotypes) } \\
\text { EUROPEAN AMERICANS }\end{array}$} & \multicolumn{2}{|c|}{$\begin{array}{c}\text { ALBUMINURIA (N Cases = } \\
\text { Cases with Enh2 TT } \\
\text { Genotype/All Cases) }\end{array}$} & \multicolumn{2}{|c|}{$\begin{array}{c}\text { MICROALBUMINURIA (N } \\
\text { Cases = Cases with Enh2 TT } \\
\text { Genotype/All Cases) }\end{array}$} & \multicolumn{2}{|c|}{$\begin{array}{c}\text { MACROALBUMINURIA (N } \\
\text { Cases = Cases with Enh2 TT } \\
\text { Genotype/All Cases) }\end{array}$} \\
\hline & OR $(95 \% \mathrm{Cl})$ & P-Value & OR $(95 \% \mathrm{Cl})$ & P-Value & OR $(95 \% \mathrm{Cl})$ & P-Value \\
\hline TYPE 2 DIABETES $(\mathrm{N}=72 / 1,095)$ & $(\mathrm{N}$ Cases $=20 / 187)$ & & $(\mathrm{N}$ Cases $=14 / 134)$ & & $(\mathrm{N}$ Cases $=6 / 53)$ & \\
\hline Model 1 & $2.11(1.19-3.73) *$ & 0.010 & $2.01(1.06-3.82) *$ & 0.034 & $2.69(1.02-7.09) *$ & 0.045 \\
\hline Model 2 & $2.14(1.20-3.80) * *$ & 0.009 & $2.00(1.05-3.81) *$ & 0.035 & $2.66(0.98-7.26)$ & 0.056 \\
\hline Model 2 + Fasting Glucose ${ }^{\S}$ & $1.99(1.07-3.68) *$ & 0.029 & $2.07(1.06-4.06) *$ & 0.034 & $1.85(0.58-5.91)$ & 0.299 \\
\hline NON-DIABETIC $(\mathrm{N}=478 / 7,027)$ & $(\mathrm{N}$ Cases $=24 / 344)$ & & (N Cases $=19 / 298)$ & & $(\mathrm{N}$ Cases $=5 / 46)$ & \\
\hline Model 1 & $1.01(0.65-1.55)$ & 0.980 & $0.91(0.56-1.48)$ & 0.713 & $1.64(0.64-4.25)$ & 0.305 \\
\hline Model 2 & $1.03(0.67-1.59)$ & 0.899 & $0.93(0.57-1.50)$ & 0.764 & $1.77(0.67-4.64)$ & 0.246 \\
\hline Model $2+$ Fasting Glucose ${ }^{\S}$ & $1.05(0.68-1.63)$ & 0.815 & $0.95(0.59-1.54)$ & 0.843 & $1.78(0.68-4.66)$ & 0.242 \\
\hline AFRICAN AMERCIANS & OR $(95 \% \mathrm{Cl})$ & P-Value & OR $(95 \% \mathrm{Cl})$ & P-Value & & \\
\hline TYPE 2 DIABETES $(\mathrm{N}=2 / 573)$ & $(\mathrm{N}$ Cases $=1 / 160)$ & & $(\mathrm{N}$ Cases $=1 / 107)$ & & $(\mathrm{N}$ Cases $=0 / 53)$ & \\
\hline Model 1 & $3.09(0.18-54.14)$ & 0.440 & $5.37(0.32-90.91)$ & 0.244 & & \\
\hline Model 2 & $4.36(0.24-79.81)$ & 0.321 & $6.39(0.37-110.75)$ & 0.203 & & \\
\hline Model $2+$ Fasting Glucose $\mathrm{s}^{\S}$ & $7.26(0.38-137.93)$ & 0.187 & $8.12(0.44-148.09)$ & 0.158 & & \\
\hline NON-DIABETIC $(\mathrm{N}=4 / 1583)$ & $(\mathrm{N}$ Cases $=1 / 134)$ & & $(\mathrm{N}$ Cases $=1 / 113)$ & & $(\mathrm{N}$ Cases $=0 / 21)$ & \\
\hline Model 1 & $3.29(0.33-32.84)$ & 0.310 & $4.14(0.41-41.44)$ & 0.227 & & \\
\hline Model 2 & $4.69(0.47-46.58)$ & 0.187 & $5.15(0.51-51.66)$ & 0.164 & & \\
\hline Model 2 + Fasting Glucose & $8.07(0.70-93.63)$ & 0.095 & $8.60(0.73-100.97)$ & 0.087 & & \\
\hline
\end{tabular}

Statistical significance of point estimates: ${ }^{*} \mathrm{P}<\left..05\right|^{* *} \mathrm{P}<\left..01\right|^{* * *} \mathrm{P}<.001$.

${ }^{\dagger}$ Analyses with microalbuminuria as the outcome excluded macroalbuminuria cases. Analyses with macroalbuminuria as the outcome excluded microalbuminuria cases. Among African Americans, analyses with macroalbuminuria were omitted due to extremely low allele frequency.

Model 1 includes age, sex, systolic blood pressure, diastolic blood pressure and hypertension medication use.

Model 2 includes Model 1 covariates and BMI and estimated glomerular filtration rate.

${ }^{\S}$ Model $2+$ Fasting Glucose is limited to individuals with fasting glucose values: non-diabetic European Americans $\mathrm{N}=6,927$ (340 albuminuria cases) and European Americans with type 2 diabetes $\mathrm{N}=983$ (166 albuminuria cases) and non-diabetic blacks $\mathrm{N}=1,520$ (126 albuminuria cases) and blacks with type 2 diabetes $\mathrm{N}=415$ (100 albuminuria cases).

Among European Americans, P-values estimating interaction between GLUT Enh2 and diabetes were not significant for the outcomes of albuminuria $(P$-interaction $=0.064)$, microalbuminuria $(P$-interaction $=0.071)$, and macroalbuminuria $(P$-interaction $=0.730)$. Among blacks, $P$-values estimating interaction between GLUT Enh2 and diabetes were not significant for the outcomes of albuminuria $(P$-interaction $=0.86)$, microalbuminuria $(P$-interaction $=0.96)$. 
Table 5 Unadjusted and multivariate linear regression of In (ACR) and serum creatinine for Enh 2 in all genotyped European Americans, by type 2 diabetes status

\begin{tabular}{|c|c|c|c|c|}
\hline \multirow[b]{2}{*}{ (N = Enh2 TT Genotype/All Genotypes) } & \multicolumn{4}{|c|}{ Regression coefficient forGLUT1 Enh2 Risk Genotype(TT) $(95 \% \mathrm{Cl})$ reference: (CC or CT) } \\
\hline & In (ACR) B Coefficient (95\% Cl) & P-value & Serum Creatinine B Coefficient $(95 \% \mathrm{Cl})$ & P-value \\
\hline \multicolumn{5}{|l|}{ TYPE 2 DIABETES $(\mathrm{N}=72 / 1,095)$} \\
\hline Unadjusted & $0.26(-0.17-0.69)$ & 0.240 & $0.06(-0.00-0.13)$ & 0.069 \\
\hline Model 1 & $0.27(-0.14-0.68)$ & 0.196 & $0.02(-0.04-0.09)$ & 0.446 \\
\hline Model 2 & $0.28(-0.13-0.68)$ & 0.185 & $0.02(-0.04-0.09)$ & 0.446 \\
\hline \multicolumn{5}{|l|}{ NON-DIABETIC $(\mathrm{N}=478 / 7,027)$} \\
\hline Unadjusted & $-0.02(-0.12-0.09)$ & 0.737 & $-0.01(-0.04-0.01)$ & 0.189 \\
\hline Model 1 & $-0.02(-0.12-0.08)$ & 0.702 & $-0.01(-0.03-0.01)$ & 0.146 \\
\hline Model 2 & $-0.02(-0.12-0.08)$ & 0.667 & $-0.01(-0.03-0.00)$ & 0.143 \\
\hline
\end{tabular}

Model 1 includes age, sex, systolic blood pressure, diastolic blood pressure and hypertension medication use.

Model 2 includes Model 1 covariates and BMI and estimated glomerular filtration rate for the outcome In (ACR). For the outcome of serum creatinine, model 2 includes Model 1 covariates and BMI.

\section{The GLUT1 Enh2 Risk Genotype and Insulin}

To examine effects of insulin concentration on the association of GLUT1 Enh2 genotypes and albuminuria, we stratified by insulin concentrations excluding those with diabetes (to avoid confounding due to insulin treatment for diabetes). Due to the low frequency of the GLUT1 Enh2 risk genotype among African Americans, analysis was limited to European Americans. Among European Americans, ( $n=6583$ controls, 294 cases of microalbuminuria, and 46 cases of macroalbuminuria), the mean fasting insulin concentration was $10.9 \mu \mathrm{U} / \mathrm{mL}$ (S.D. = 7.5). Risk of albuminuria for Enh2 TT was OR 1.08 (95\% CI: 0.70 - 1.67, p = 0.724), adjusting for age, gender, hypertension status, BMI, and GFR. Among individuals with insulin in the highest quartile (mean insulin $19.9 \mu \mathrm{U} / \mathrm{mL}$, S.D. = 9.7), the adjusted OR of albuminuria for Enh2 TT was 1.25 (95\% CI: $0.60-2.58$, p = 0.547). Among individuals with insulin concentrations in the lower three quartiles (mean $7.9 \mu \mathrm{U} / \mathrm{mL}$, S.D. = 2.9), the adjusted OR of albuminuria was 1.00 (95\% CI: 0.58 $1.72, \mathrm{p}=0.990)$ for Enh2 TT carriers. When we focused on the more specific outcome of macroalbuminuria as a phenotype and excluded patients with microalbuminuria, risk of macroalbuminuria for Enh2 TT was OR 1.84 (95\% CI: $0.71-4.77, \mathrm{p}=0.210$ ) as illustrated in figure 2. Among individuals with insulin in the highest quartile, the adjusted OR for Enh2 TT was 4.08 (95\% CI: 1.06 - 15.61, $\mathrm{p}=0.040)$. Among individuals with insulin concentrations in the lower three quartiles, the adjusted OR of macroalbuminuria was 1.00 (95\% CI: 0.23 - 4.28; P = 0.995) for Enh2 TT carriers. Among non-diabetics, a formal test of interaction of the GLUT1 Enh2 risk genotype and the upper quartile of insulin was not significant for macroalbuminuria $(P=0.163)$.

\section{Discussion}

Genetic variation of GLUT1 may be associated with the risk of micro- and macroalbuminuria in the general U.S. adult population of European Americans with type 2 diabetes. Though an association was seen when we combined European Americans with diabetes from stages 1 and 2, the findings of stage 1 were not replicated in stage 2. Both XbaI and Enh2 SNPs appeared to be associated in a recessive fashion with albuminuria, consistent with previous studies $[5,6,8,11,13]$. Further analysis showed Enh2 risk genotype (TT) increased risk independent of all other genotyped GLUT1 SNPs, including XbaI, suggesting that Enh2 is the causative GLUT1 SNP associated with albuminuria. Addtionally, no other GLUT1 SNPs besides Enh2 have been demonstrated to be located in a region with functional activity in vitro $[5,6,8,13]$. By study stage, the Enh2 risk genotype was associated with diabetic albuminuria among European Americans only in stage 1 and in the total study population; these findings were not replicated when we examined stage 2. In the total study population of European Americans with type 2 diabetes, the risk effect of Enh2 on macroalbuminuria was independent of age, gender, and hypertension. Possession of the Enh2 risk genotype and high insulin concentrations particularly increased macroalbuminuria risk among non-diabetic European Americans. Among African Americans, XbaI was not associated with albuminuria. The association of the Enh2 risk genotype and albuminuria among African Americans was increased among individuals with and without type 2 diabetes but was not statistically significant. Despite having over 2000 African Americans in the present study, the low frequency of the Enh2 risk genotype in this population $(0.3 \%)$ limited the power of our analysis and prevents us from making definitive conclusions about the role of GLUT1 genetic variation and albuminuria in African Americans. However, our results suggest that GLUT1 Enh2 may be associated with albuminuria.

In European Americans, our results extend the findings of a prior study by $\mathrm{Ng}$ et al. among type 1 diabetics 


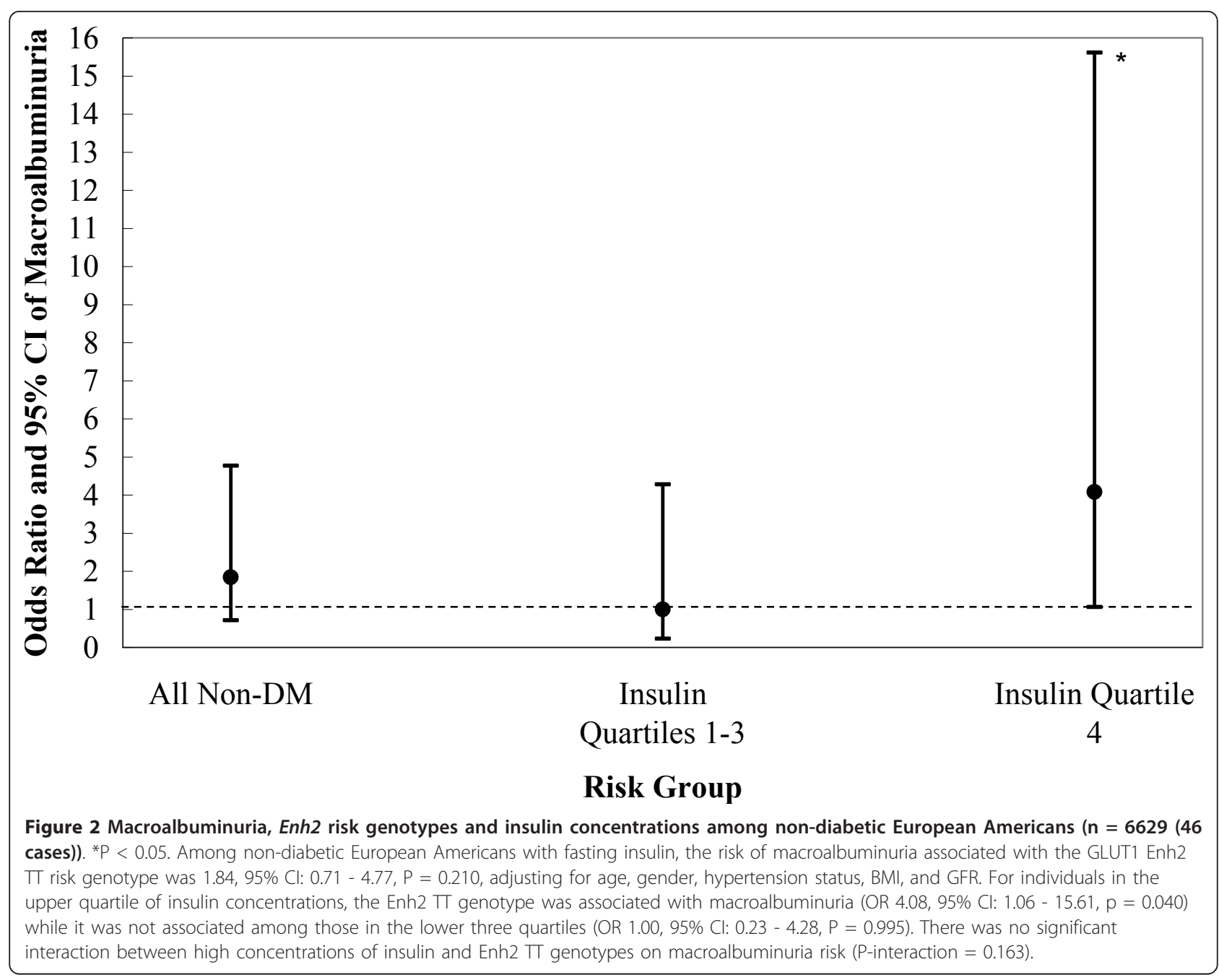

[35], suggesting both XbaI and Enh2 homozygotes as having an increased risk of albuminuria. However, the authors could not examine the independent risk effect of Enh2 or XbaI separately; since no individuals in their study possessed the Enh2 risk genotype but not the XbaI risk genotype. Multivariate analysis in our study suggest the XbaI AA risk genotype by itself does not confer risk $(\mathrm{OR}=0.94, \mathrm{p}=0.931)$, whereas the Enh2 $\mathrm{TT}$ risk genotype $(\mathrm{OR}=3.37, \mathrm{p}=0.090)$ has a stronger association with albuminuria independent of XbaI. Our results extend the risk association of Enh2 to albuminuria in European Americans with type 2 diabetes. Additionally, a recent study in Tunisia of type 2 diabetics had similarly demonstrated an association of the Enh2 TT risk genotype with nephropathy (OR 8.4 (95\% CI: 3.3-21.5)) while finding no association with $\mathrm{XbaI}$ [11].

Additionally, our results suggest that among non-diabetic European Americans with the Enh2 risk genotype, those with higher concentrations of insulin were at greater risk of having proteinuria. The putative human enhancer-2 is homologous with the murine enhancer-2 which was responsive to insulin in vitro $[5,24]$. Furthermore, the Enh2 SNP is located within a binding site for the insulin-responsive transcription factor, the upstream stimulatory factor (USF) $[5,25,26]$. USF has been identified as a glucose-inducible transcription factor in mesangial cells $[27,28]$ and shown to regulate GLUT1 transcription [29]. The Enh2 SNP of GLUT1 is located within a USF responsive element in humans, suggesting the TT genotype might have altered gene expression of GLUT1 that contributes to diabetic nephropathy [36]. Additionally, the interaction of the Enh2 SNP and insulin supports the previously posited hypothesis [5] that in individuals with the Enh2 risk genotype, high intracellular glucose concentrations might increase in mesangial cells in response to insulin [37]. The high concentrations of intracellular glucose may contribute to mesangial matrix expansion and glomerulosclerosis through several pathologic cellular mechanisms including the 
polyol pathway, activation of protein kinase $C$, increased formation of advanced glycation end-products, and the hexosamine pathway $[38,39]$. Additionally, there is evidence that podocytes are critical in maintaining the glomerular filtration barrier of the kidney and preventing albuminuria [40-42], and a recent study demonstrated that the glucose uptake of podocytes are insulin responsive and act via GLUT1, suggesting the insulin sensitivity of human podocytes resulting in urinary protein loss may act via these mechanisms [43]. Future studies should examine if the Enh2 SNP indeed modulates GLUT1 protein expression. Furthermore, most candidate gene chips (Affymetrix 5.0, 6.0, 500K, 100K; Illumina 550K, 650K) do not include Enh2 SNP. This suggests follow-up studies may need de-novo genotyping.

Previous studies of $\mathrm{XbaI}$ and diabetic nephropathy have had disparate results [12]. Our results for the XbaI risk genotype in European Americans are consistent with previous studies which demonstrated increased risk of type 1 diabetic nephropathy among those homozygous for the XbaI A allele [5,6], suggesting recessive transmission of the phenotype. Inconsistent results in other studies of XbaI may be due to different patterns of disequilibrium with the Enh2 polymorphism [7-11].

Our study has several limitations. Its design is crosssectional and the albuminuria classification is based on a spot urine ACR because urine which was only collected at one ARIC visit. A phenotype based on a single measurement of ACR could definitely lead to incorrect conclusions, particularly with potential misclassification of borderline values with a dichotomous outcome such as albuminuria. However, sensitivity analyses for macroalbuminuria demonstrated a significant risk association with the Enh2 risk genotype. Another limitation is that the Enh2 risk genotype was only significantly associated with diabetic albuminuria among European Americans from stage 1 and in the combined analysis. The findings of stage 1 were not replicated in stage 2 . The samples from stage 1 and 2 were not randomly sampled, with stage 1 consisting of a convenience sample enriched for those with type 2 diabetes and African Americans, a sample previously used for the study of genetic risk factors in cardiovascular disease and type 2 diabetes. Participants comprising stage 2 consisted of the remaining ARIC visit 4 particpants not in sample 1. However, Enh2 TT still had an increased (but not statistically significant) association with albuminuria among European Americans with diabetes from stage 2, and had a significant and strong association with diabetic albuminuria when we combined both stages. Additionally, despite the fact that our study included 8122 European Americans, of whom there were 1095 type 2 diabetics, given the rarity of the Enh2 TT risk genotype among diabetics $(6.6 \%)$, the size of our study precludes any definitive conclusions regarding the association of type 2 diabetic albuminuria and the Enh2 polymorphism of GLUT1. Given this limitation, our study can at best suggest an association between Enh2 and albuminuria among European American type 2 diabetics; however, due to the limitation in our number of subjects, we do not have sufficient power to make conclusive statements regarding this association. Perhaps a larger study of type 2 diabetic European Americans involving multiple cohorts may provide more power to definitively evaluate the role of the Enh2 risk genotype of GLUT1 and albuminuria. Furthermore, we did not have hemoglobin A1C (HbA1C) for all participants in our sample, though in our sub-sample, adjustment did not significantly affect results. Instead, we used fasting glucose as a marker of glycemic control. However, $\mathrm{Ng}$ et al. had previously found the strength of the association of the Enh2 risk genotype and diabetic albuminuria was independent of both $\mathrm{HbA1C}$ and duration of diabetes [5]. Ours is the largest study of GLUT1 genetic variation and albuminuria to date and the first to examine associations among non-diabetics and African Americans. We substantiate prievous findings and extend the association beyond type 1 diabetes, demonstrating a role for GLUT1 Enh2 and proteinuria among those with type 2 diabetes.

\section{Conclusions}

In summary, GLUT1 genetic variation of Enh2 may predict risk of micro- and macroalbuminuria among European Americans with type 2 diabetes. Though the Enh2 risk genotype was significantly associated with diabetic albuminuria among European Americans from stage 1 and in the combined analysis, the findings of stage 1 were not replicated in stage 2 . Furthermore, the rarity of the Enh2 risk genotype among African Americans precludes any definitive conclusions, although data suggest a risk-enhancing role. Our results suggest the Enh2 SNP, and not XbaI, is the causative polymorphism associated with diabetic albuminuria. Additionally, the Enh2 risk genotype may interact with hyperinsulinemia to further increase susceptibility to albuminuria, consistent with hypotheses generated by laboratory data. Studying mechanisms mediating this association may shed light on novel pathways and therapeutic targets in the pathophysiology of albuminuria and nephropathy. The modest size of the risk associated with GLUT1 Enh2 variation limits utility for screening, risk stratification and individualized therapy. However, if multiple genes of small and moderate effect on nephropathy are identified, they may compose panels for risk assessment.

\section{Acknowledgements}

The Atherosclerosis Risk in Communities Study is carried out as a collaborative study supported by National Heart, Lung, and Blood Institute 
contracts N01-HC-55015, N01-HC-55016, N01-HC-55018, N01-HC-55019, N01 HC-55020, N01-HC-55021, and N01-HC-55022. The American Diabetes Association and National Kidney Foundation of Maryland also provided support for C.C.H. The authors thank the staff and participants of the ARIC study for their important contributions.

\section{Author details}

'Departments of Medicine and Epidemiology, Johns Hopkins Medical Institutions, Baltimore, Maryland, USA. ${ }^{2}$ Department of Laboratory Medicine and Pathology, University of Minnesota, Minneapolis, Minnesota, USA. ${ }^{3}$ Department of Medicine, University of Maryland School of Medicine, Baltimore, Maryland, USA. ${ }^{4}$ Human Genetics Center, University of TexasHouston Health Science Center, Houston, Texas, USA. ${ }^{5}$ Department of Medicine, University of Chicago, Chicago, Illinois, USA. ${ }^{6}$ Department of Radiation Oncology, University of California, San Francisco, California, USA.

\section{Authors' contributions}

$\mathrm{CCH}$, WHLK, CWH, and JC designed the study. Laboratory work was undertaken by MWS, WHLK, TG, FLB, EB, and AS. Statistical analysis was done by $\mathrm{CCH}, \mathrm{WHLK}$, and JC. CCH wrote the first draft of the paper. All authors contributed to and approved of the final version of the manuscript.

\section{Competing interests}

The authors declare that they have no competing interests.

Received: 29 September 2009 Accepted: 19 January 2011 Published: 19 January 2011

\section{References}

1. Heilig C, Zaloga C, Lee M, Zhao X, Riser B, Brosius F, et al: Immunogold localization of high-affinity glucose transporter isoforms in normal rat kidney. Lab Invest 1995, 73:674-84.

2. Heilig CW, Liu Y, England RL, Freytag SO, Gilbert JD, Heilig KO, et al: Dglucose stimulates mesangial cell GLUT1 expression and basal and IGF-Isensitive glucose uptake in rat mesangial cells: implications for diabetic nephropathy. Diabetes 1997, 46:1030-9.

3. Heilig CW, Concepcion LA, Riser BL, Freytag SO, Zhu M, Cortes P. Overexpression of glucose transporters in rat mesangial cells cultured in a normal glucose milieu mimics the diabetic phenotype. $J$ Clin Invest 1995, 96:1802-14.

4. Gnudi L, Viberti G, Raij L, Rodriguez V, Burt D, Cortes P, et al: GLUT-1 overexpression: Link between hemodynamic and metabolic factors in glomerular injury? Hypertension 2003, 42:19-24.

5. Ng DP, Canani L, Araki S, Smiles A, Moczulski D, Warram JH, et al: Minor effect of GLUT1 polymorphisms on susceptibility to diabetic nephropathy in type 1 diabetes. Diabetes 2002, 51:2264-9.

6. Hodgkinson AD, Millward BA, Demaine AG: Polymorphisms of the glucose transporter (GLUT1) gene are associated with diabetic nephropathy. Kidney Int 2001, 59:985-9.

7. Liu ZH, Guan TJ, Chen ZH, Li LS: Glucose transporter (GLUT1) allele (Xbal-) associated with nephropathy in non-insulin-dependent diabetes mellitus. Kidney Int 1999, 55:1843-8.

8. Grzeszczak W, Moczulski DK, Zychma M, Zukowska-Szczechowska E, Trautsolt W, Szydlowska I: Role of GLUT1 gene in susceptibility to diabetic nephropathy in type 2 diabetes. Kidney Int 2001, 59:631-6.

9. Tarnow L, Grarup N, Hansen T, Parving HH, Pedersen O: Diabetic microvascular complications are not associated with two polymorphisms in the GLUT-1 and PC-1 genes regulating glucose metabolism in Caucasian type 1 diabetic patients. Nephrol Dial Transplant 2001, 16:1653-6

10. Gutierrez C, Vendrell J, Pastor R, Broch M, Aguilar C, Llor C, et al: GLUT1 gene polymorphism in non-insulin-dependent diabetes mellitus: genetic susceptibility relationship with cardiovascular risk factors and microangiopathic complications in a Mediterranean population. Diabetes Res Clin Pract 1998, 41:113-20.

11. Makni K, Jarraya F, Rebai M, Mnif F, Boudawara M, Hamza N, et al: Risk genotypes and haplotypes of the GLUT1 gene for type 2 diabetic nephropathy in the Tunisian population. Ann Hum Biol 2008, 35:490-498,

12. Zintzaras E, Stefanidis I: Association between the GLUT1 gene polymorphism and the risk of diabetic nephropathy: a meta-analysis. $J$ Hum Genet 2005, 50:84-91.
13. Hodgkinson AD, Page T, Millward BA, Demaine AG: A novel polymorphism in the $5^{\prime}$ flanking region of the glucose transporter (GLUT1) gene is strongly associated with diabetic nephropathy in patients with Type 1 diabetes mellitus. J Diabetes Complications 2005, 19:65-69.

14. The Atherosclerosis Risk in Communities (ARIC) Study: design and objectives. The ARIC investigators. Am J Epidemiol 1989, 129:687-702.

15. National Heart LaBI: In ARIC Manual of Operations: no. 1 general description and study management; no 2 cohort component procedures; no 3 surveillance component procedures; no. 4 pulmonary function assessment; no. 5 electrocardiography; no. 6 ultrasound assessment; no. 7 blood collection and processing; no. 8 lipid and lipoprotein determinations; no. 9. Hemostasis determinations; no. 10 clinical chemistry determinations; no. 11 sitting blood pressure and postural changes in blood pressure and heart rate; no. 12 quality assurance. Volume 4. ARIC Coordinating Center, School of Public Health, University of North Carolina, Suite 203, NCNB Plaza, 137 E. Franklin St., Chapel Hilll, NC 2751; 1987.

16. Levey AS, Greene T, Kusek JW, Beck GJ, MDRD Study Group: A simplified equation to predict glomerular filtration rate from serum creatinine. $J$ Am Soc Nephrol 2000, 11:155A, (abstrA0828).

17. K/DOQI clinical practice guidelines for chronic kidney disease: evaluation classification and stratification. Am J Kidney Dis 2002, 39:51-266.

18. Molitch ME, DeFronzo RA, Franz MJ, Keane WF, Mogensen CE, Parving HH et al: Nephropathy in diabetes. Diabetes Care 2004, 27(Suppl 1):S79-S83.

19. Eknoyan G, Hostetter T, Bakris GL, Hebert L, Levey AS, Parving HH, et al: Proteinuria and other markers of chronic kidney disease: a position statement of the national kidney foundation (NKF) and the national institute of diabetes and digestive and kidney diseases (NIDDK). Am J Kidney Dis 2003, 42:617-622.

20. Murakami T, Nishiyama T, Shirotani T, Shinohara Y, Kan M, Ishii K, et al: Identification of two enhancer elements in the gene encoding the type 1 glucose transporter from the mouse which are responsive to serum, growth factor, and oncogenes. J Biol Chem 1992, 267:9300-9306.

21. Behrooz A, Ismail-Beigi F: Dual control of glut1 glucose transporter gene expression by hypoxia and by inhibition of oxidative phosphorylation. $J$ Biol Chem 1997, 272:5555-5562.

22. Wasson J, Skolnick G, Love-Gregory L, Permutt MA: Assessing allele frequencies of single nucleotide polymorphisms in DNA pools by pyrosequencing technology. Biotechniques 2002, 32:1144-6, 1148, 1150

23. Steinle NI, Kazlauskaite R, Imumorin IG, Hsueh WC, Pollin TI, O'Connell JR, Mitchell BD, Shuldiner AR: Variation in the Lamin A/C Gene: Associations With Metabolic Syndrome. Arterioscler Thromb Vasc Biol 2004, 24:1708-1713.

24. Todaka M, Nishiyama T, Murakami T, Saito S, Ito K, Kanai F, et al: The role of insulin in activation of two enhancers in the mouse GLUT1 gene. J Biol Chem 1994, 269:29265-29270.

25. Travers MT, Vallance AJ, Gourlay HT, Gill CA, Klein I, Bottema CB, et al: Promoter I of the ovine acetyl-CoA carboxylase-alpha gene: an E-box motif at -114 in the proximal promoter binds upstream stimulatory factor (USF)-1 and USF-2 and acts as an insulin-response sequence in differentiating adipocytes. Biochem J 2001, 359:273-284.

26. Lefrancois-Martinez AM, Martinez A, Antoine B, Raymondjean M, Kahn A: Upstream stimulatory factor proteins are major components of the glucose response complex of the L-type pyruvate kinase gene promoter. J Biol Chem 1995, 270:2640-2643.

27. Xiang M: USF2a regulation of mesangial cell (MC) GLUT1 transcription: Implications for diabetic nephropathy [abstract]. J Am Soc Nephrol 2004, 15.

28. Xiang M: Upstream stimulatory factor (USF) involvement in mesangial cell gene regulation: Implications for diabetic nephropathy [abstract]. J Am Soc Nephrol 2003, 14.

29. Heilig CW, Brosius FC III, Cunningham C: Role for GLUT1 in diabetic glomerulosclerosis. Expert Rev Mol Med 2006, 8:1-18.

30. Rice TK, Schork NJ, Rao DC: Methods for handling multiple testing. Adv Genet 2008, 60:293-308

31. Stephens M, Donnelly P: A comparison of bayesian methods for haplotype reconstruction from population genotype data. Am J Hum Genet 2003, 73:1162-1169.

32. Stephens M, Smith NJ, Donnelly P: A new statistical method for haplotype reconstruction from population data. Am J Hum Genet 2001, 68:978-989.

33. Devlin B, Risch N: A comparison of linkage disequilibrium measures for fine-scale mapping. Genomics 1995, 29:311-322. 
34. Lewontin RC: The interaction of selection and linkage. ii. Optimum models. Genetics 1964, 50:757-782.

35. Ng DP, Canani L, Araki S, Smiles A, Moczulski D, Warram JH, et al: Minor effect of GLUT1 polymorphisms on susceptibility to diabetic nephropathy in type 1 diabetes. Diabetes 2002, 51:2264-2269.

36. Heilig KO, Chen S, Xiang M, Brosius FC, Heilig CW: Overexpression of GLUT1 in glomeruli produces features of diabetic nephropathy in mice [abstract]. J Am Soc Nephrol 2004, 15.

37. Arnqvist HJ, Ballermann BJ, King GL: Receptors for and effects of insulin and IGF-I in rat glomerular mesangial cells. Am J Physiol 1988, 254: C411-C416.

38. Brownlee M: Biochemistry and molecular cell biology of diabetic complications. Nature 2001, 414:813-820.

39. Henry DN, Busik JV, Brosius FC3, Heilig CW: Glucose transporters control gene expression of aldose reductase, PKCalpha, and GLUT1 in mesangial cells in vitro. Am J Physiol 1999, 277:97-104.

40. Kestila M, Lenkkeri U, Mannikko M, Lamerdin J, McCready P, Putaala H, et al: Positionally cloned gene for a novel glomerular protein-nephrin-is mutated in congenital nephrotic syndrome. Mol Cell 1998, 1:575-582.

41. Boute N, Gribouval O, Roselli S, Benessy F, Lee H, Fuchshuber A, et al: NPHS2, encoding the glomerular protein podocin, is mutated in autosomal recessive steroid-resistant nephrotic syndrome. Nat Genet 2000, 24:349-354.

42. Kaplan JM, Kim SH, North KN, Rennke H, Correia LA, Tong HQ, et al: Mutations in ACTN4, encoding alpha-actinin-4, cause familial focal segmental glomerulosclerosis. Nat Genet 2000, 24:251-256.

43. Coward RJ, Welsh Gl, Yang J, Tasman C, Lennon R, Koziell A, et al: The human glomerular podocyte is a novel target for insulin action. Diabetes 2005, 54:3095-3102.

\section{Pre-publication history}

The pre-publication history for this paper can be accessed here: http://www.biomedcentral.com/1471-2350/12/16/prepub

doi:10.1186/1471-2350-12-16

Cite this article as: Hsu et al: Genetic variation of Glucose Transporter-1 (GLUT1) and albuminuria in 10,278 European Americans and African Americans: a case-control study in the Atherosclerosis Risk in Communities (ARIC) Study. BMC Medical Genetics 2011 12:16.

\section{Submit your next manuscript to BioMed Central and take full advantage of:}

- Convenient online submission

- Thorough peer review

- No space constraints or color figure charges

- Immediate publication on acceptance

- Inclusion in PubMed, CAS, Scopus and Google Scholar

- Research which is freely available for redistribution

Submit your manuscript at www.biomedcentral.com/submit 